Scientific Paper

\title{
Comparative study between Acuros XB algorithm and Anisotropic Analytical Algorithm in the case of heterogeneity for the treatment of lung cancer
}

\author{
Mohammed El Adnani KRABCH ${ }^{1,2, a}$, Abdelouahed CHETAINE ${ }^{1}$, Abdelati NOURREDDINE ${ }^{1}$, \\ Fatim Zohra ER-RADI ${ }^{1}$, Laila BADDOUH ${ }^{1}$ \\ ${ }^{I}$ Nuclear reactor, nuclear security and environment group, Physics Department, Faculty of Sciences, \\ Mohamed V University, Rabat, Morocco \\ ${ }^{2}$ Sheikh Khalifa Ibn Zaid Hospital Department of Radiotherapy, Casablanca, Morocco. \\ ${ }^{a}$ E-mail address: krabch.adnani@gmail.com
}

(received 24 January 2018; revised 27 March 2018; accepted 1 August 2018)

\begin{abstract}
The aim of this study was to investigate the impact of heterogeneity on the dose calculation for two algorithms implemented in the TPS "Analytical Anisotropic Algorithm (AAA) and Acuros XB" and validated the use of Acuros $\mathrm{XB}$ algorithm in clinical routine. First, we compare the dose calculated by these algorithms and the dose measured at the given point $\mathrm{P}$, which is found after heterogeneity insert. Second, we extend our work on clinical cases that present a complex heterogeneity. By evaluating the impact of the choice of the algorithm on the dose coverage of the tumor, and the dose received by the organs at risk for 20 patients affected by lung cancer.

The result of our phantom study showed a good agreement with several studies that showed the superiority of the Acuros XB over the AAA in predicting dose when it concerns heterogeneous media. The treatment plans for 20 lung cancers were calculated by two algorithms AAA and Acuros XB, the results show a statistical significant difference between algorithms for Homogeneity Index and the maximum dose of planning target volume (HI: $0.11 \pm 0.01$ vs $0.05 \pm 0.01 \mathrm{p}=0.04$; Dmax: $69.30 \pm 3.12$ vs $68.51 \pm 2.64 \mathrm{p}=0.02)$. Instead, no statistically significant difference was observed for conformity index CI and mean dose (CI: $0.98 \pm 0.18$ vs $0.99 \pm 0.14 \mathrm{p}=0.33$; Dmean: $66.3 \pm 0.65$ vs 66.10 $\pm 0.61 \mathrm{p}=0.54$ ). For organs at risk, the maximum dose for spinal cord, mean dose and D37 \% of lung minus GTV (dose receiving $37 \%$ of lung volume) were found to be lower for AAA plans than Acuros XB and the differences were statistically significant $(\mathrm{p}<0.05)$. For the heart D33\% and D67\% were found to be higher for AAA plans than Acuros $\mathrm{XB}$ and the differences were statistically significant $(\mathrm{p}<0.05)$, but No difference was observed for D100\% of the heart. The use of the AXB algorithm is suitable in the case of presence of heterogeneity, because it allows to have a better accuracy close to the Monte Carlo calculation.
\end{abstract}

Key words: lung cancer; Analytical Anisotropic Algorithm; Acuros XB; TPS.

\section{Introduction}

The goal of external radiotherapy is to deliver a sufficiently high dose of ionizing radiation to the tumor whilst preserving organs at risk and healthy tissues. In order to achieve this goal sophisticated algorithms for dose distribution calculation implemented in computerized treatment planning systems are used.

The algorithms used in the 50s were 2D algorithms based on the tissue-air ratio (TAR). Also other simple method analytical methods of radiation transport were used. According to these algorithms the dose below an inhomogeneity was calculated assuming that the inhomogeneity of density $\rho i$ and thickness $\Delta \mathrm{di}$ attenuates the beam as the equivalent slab of water of thickness $\Delta \mathrm{di} \cdot \rho \mathrm{w}$.
The correction factor, which is the ratio of dose in the actual (inhomogenous one) geometry and homogenous one was given by the formulae:

$C F=\frac{\operatorname{TAR}\left(\mathrm{d}^{\prime}, \mathrm{Sd}\right)}{\operatorname{TAR}(\mathrm{d}, \mathrm{Sd})}$

Eq. 1

Where the numerator is the TAR for the equivalent water thickness, $d$ ' which given by equation:

$d^{\prime}=\sum_{i}(\Delta \mathrm{di} \cdot \rho \mathrm{i})$

Eq. 2

and the denominator is the TAR for the physical depth of the point of interest. Sd is the size of the beam at the level of the point of interest. The major weakness of the TAR method is its approximate modeling of the lateral component of the scattered photon contribution that result in an over-correction when the density is less than that of water and an under-correction when 
the density is greater than water. Considering the evolution of imaging systems and computing, new algorithms have been developed to calculate the primary beam, scattered in space in three dimensions, and taking into account the electron transport.

Dose calculation algorithms in radiotherapy treatment planning system can be classified into three groups:

Correction based: With the correction-based algorithm [1-4], dose distribution is calculated in water as a homogeneous medium that is water and corrected to account the influence of irregular shape of the patient's surface and the heterogeneity in the human body. The density variation is taken into account for the primary radiation (directly from the source to the point of interest) but not for the scattered radiation (radiation not coming from the source), the changes of the lateral transport of the electrons are not taken into account [5]. This method overestimates the dose in the presence of low density and underestimates it in the presence of high density, but the medical physicist still uses it to obtain an approximate result quickly or for a double calculation [6].

Convolution model algorithm is introduced in the modern treatment planning systems TPS. This method is based on the convolution of the energy distribution due to the primary particles with a "kernel" describing the dose distribution by secondary particles and the effects of the presence of heterogeneities are taken into account. There are two classes of convolution algorithm:

- Kernel pencil-beam algorithm: this algorithm assumes that the kernel remains invariant and the algorithm calculates the dose distribution without taking into account the changes in the lateral transport of electrons, Pencil Beam Convolution (PBC) is an example of this algorithm [7-11].

- Point-spread functions: this algorithm assumes that the kernel is deformed according to the electron density around it at the point of interaction. It takes into account the lateral transport of electrons approximately in the presence of heterogeneity; this is the case of the Collapsed Cone Convolution (CCC) algorithm of Pinnacle TPS (Phillips) [12-14] or AAA of Eclipse TPS (Varian) [15-19].

Algorithms based on "physical principles" as for example Acuros XB algorithm [20], is a deterministic algorithm that is based on the approximations of the Boltzman equation solving methods. It takes into account the lateral transport of electrons and the effect of heterogeneity on the dose calculation, the algorithm uses the Fokker-Planck equation (describing the spatially and temporally evolution of the probability density of a type of particles) to solve the particle transport. The computer resolution of the problem can be done very quickly and close to the Monte Carlo calculation.

Our purpose in this study is to evaluate the accuracy of the dose calculation performed with two different algorithms implemented in the TPS Analytical Anisotropic Algorithm
(AAA) and Acuros $\mathrm{XB}$, and to validate the use of Acuros $\mathrm{XB}$ algorithm in clinical routine.

\section{Materials and Methods}

In this study, we compare the doses calculated with two algorithms, namely Analytical Anisotropic Algorithm (AAA) and Acuros XB algorithm installed in treatment planning system (TPS) Eclipse version 13.5. Calculations were performed for a variety of geometries proposed by the IAEA recommendations [21]. We also compared results of calculations with measurements. All measurements presented here were carried out for $6 \mathrm{MV}$ photon beams (QI: 0.665 ) produced by Varian Truebeam STX linear accelerator, equipped with a Millennium 120 MLC HD (Varian Medical Systems, Palo Alto, CA) that offers $0.25 \mathrm{~cm}$ resolution at isocenter for the central region. Each side of the Varian HD120 MLC is configured with 60 leaves distributed in a $8 \mathrm{~cm}$ wide central region with $32 \times 2.5 \mathrm{~mm}$ leaves, flanked by two $7 \mathrm{~cm}$ wide side regions with $14 \times 5.0 \mathrm{~mm}$ leaves, for a total width of $22 \mathrm{~cm}$.

For the experimental set up a new phantom was created. It is made of Plastic Water and contains several inserts $(12 \times 12 \times 3$ $\mathrm{cm}^{3}$ ) with different tissue equivalent electron density (Table 1) at $2 \mathrm{~cm}$ of depth, and the phantom can accommodate any ion chamber for dose measurements (Figure 1).

The measurements were performed by delivering 100 monitor units (MUs) at the central axis depth doses at point $\mathrm{P}$ for an open field size $10 \times 10 \mathrm{~cm}^{2}$ by using a cylindrical chamber of 0.125 cc collecting volume (model 31010 Semiflex: PTW).

Table 1. Physical property of different inserts

\begin{tabular}{lcc}
\hline \hline Insert & $\begin{array}{c}\text { Physical Density } \\
\text { g/cc }\end{array}$ & $\begin{array}{c}\text { Electron Density } \\
\mathbf{x ~ 1 0} \mathbf{1 0}^{\mathbf{2 3}} \text { electrons/cc }\end{array}$ \\
\hline Lung + (Exhale) & 0.507 & 1.632 \\
Lung - (Inhale) & 0.205 & 0.634 \\
Bone + (Solide Dense Bone) & 1.53 & 4.862 \\
Bone - (Solide Trabecular Bone) & 1.16 & 3.730 \\
Muscle & 1.06 & 3.483 \\
Water & 1 & 3.340 \\
\hline \hline
\end{tabular}

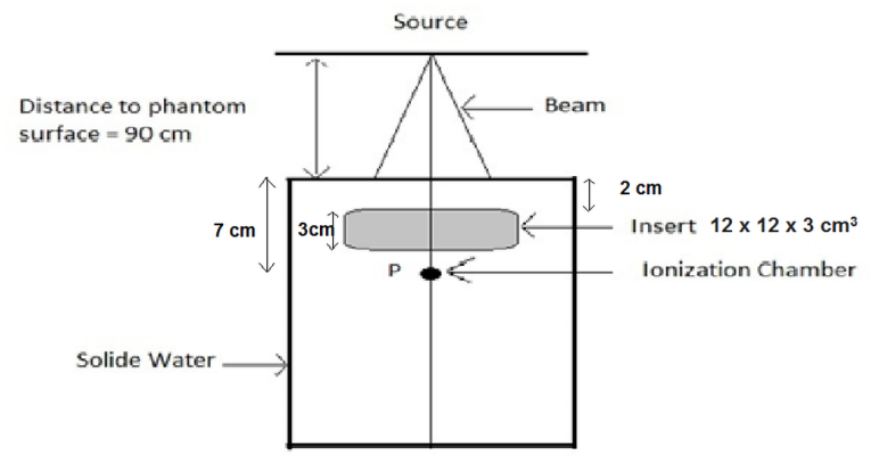

Figure 1. Schematic of experimental set-up 
The CT scans of phantom were acquired with 512 x 512 pixels at $0.25 \mathrm{~cm}$ slice spacing on GE LightSpeed CT Scanner and imported to Eclipse for calculating the dose by using 2 algorithms. Then we normalized the calculated dose (AAA and AXB) at dmax and compared to measurement.

Twenty-lung cancer patients were included in this study. Each patient was set up in the supine position and the upper body was immobilized with cradle with their arms overhead. Scanner images were acquired under the same conditions as the phantom and imported into the TPS. The physician contoured the target volume PTV and organs at risk such as the spinal cord, the lungs and the heart.

Treatment plans of all patients were generated using two arcs (technique: RapidArc; Energy: $6 \mathrm{MV}$ X-ray; machine: Truebeam STX) gantry angles from $181^{\circ}$ to $179^{\circ}$ and optimized by using Photon Optimizer algorithm (PO). The prescribed dose at PTV was 66 Gy with a daily dose of 2 Gy in 33 fractions. The aims of the planning were to give at least $95 \%$ of the prescribed doses to at least $95 \%$ of the PTV while minimizing the volumes irradiated to the organs at risk. At the end of the optimization, each plan was calculated by the AAA algorithm and recalculated by Acuros XB algorithm, after calculation we have normalized all plans at the mean of target volume. The grid size used for dose calculation of all plans was set to $2.5 \mathrm{~mm}$.

Treatment plans were evaluated comparing the maximum dose, the average dose, the conformity index (Equation 3) and the homogeneity index (Equation 4) for PTV. For organs at risk, this evaluation is performed by comparing the maximum dose received by the spinal cord, the dose received by $33 \%$, $67 \%$ and $100 \%$ of the heart volume, the average dose and the dose received by $37 \%$ of the volume of the healthy lungs.

The conformity index is the ratio between the reference volume $\mathrm{V}_{95 \%}$ and the volume of PTV.

$\mathrm{CI}=\left(\frac{\mathrm{V} 95 \%}{\mathrm{VPTV}}\right)$

Eq. 3

The homogeneity index is the difference between the near maximum dose $\left(\mathrm{D}_{2 \%}\right)$ and the near minimum dose $\left(\mathrm{D}_{98 \%}\right)$ normalized by the median dose.

$\mathrm{HI}=\left(\frac{\mathrm{D} 2 \%-\mathrm{D} 98 \%}{\text { mediane dose }}\right)$

Statistical analysis was performed with SPSS 20.0 statistical software (Chicago, IL, USA). The differences of various parameters between the two groups were analyzed and compared with paired two sided Student's t-test. $\mathrm{P}<0.05$ was considered statistically significant.

\section{Results}

Figure 2 shows the dose calculated from two algorithms AAA and Acuros XB, and measured dose at the Clinac by using ionization chamber. The observed difference between AAA algorithm and measurement after water, muscle, lung- (inhale), lung+ (exhale), bone+ (solide dense), and bone- (solide trabecular) inserts are respectively $2.4 \%, 1.6 \%, 3.1 \%, 2.6 \%$,
$2.2 \%, 3.6 \%$. Concerning Acuros algorithm, the differences observed with the same inserts in previous order are respectively $0.7 \%, 0 \%, 0.3 \%, 0.5 \%, 0.6 \%, 1 \%$, The results showed that the Acuros algorithm is very close to the measurements in different heterogeneity and better compared to the AAA algorithm.

When comparing the dose coverage of planning target volume calculated by TPS Eclipse from the two calculation algorithms AAA and Acuros, Table 2 show statistical significance difference between algorithms for HI and Dmax. The homogeneity index and the maximum dose were higher for AAA plans than Acuros plans (HI: $0.11 \pm 0.01$ vs $0.05 \pm 0.01 \mathrm{p}=$ 0.04; Dmax: 69.30 \pm 3.12 vs $68.51 \pm 2.64 \mathrm{p}=0.02$ ). Instead, no statistically significant difference was observed for conformity index CI and mean dose (CI: $0.97 \pm 0.18$ vs $0.99 \pm 0.14 \mathrm{p}=0.33$; Dmean: $66.3 \pm 0.65$ vs $66.10 \pm 0.61 \mathrm{p}=0.54)$.

For organs at risk, Table 3 shows the maximum dose for spinal cord, mean dose and D37 \% for lung minus GTV (dose receiving $37 \%$ of lung volume) were found to be lower for AAA plans than Acuros $\mathrm{XB}$ and the differences were statistically significant $(\mathrm{p}<0.05)$. For heart D33\% and D67\% were found to be higher for AAA plans than Acuros XB and the differences were statistically significant $(\mathrm{p}<0.05)$, but No difference was observed for D100\% of the heart.

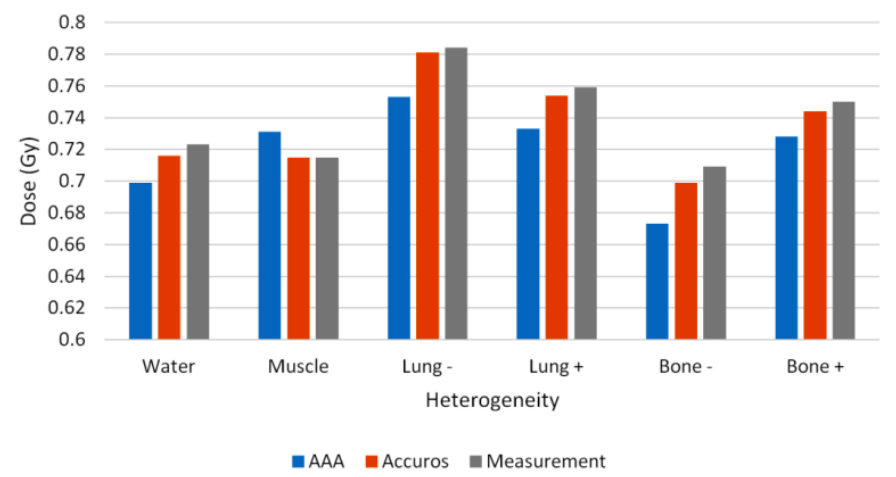

Figure 2. Dose calculated by AAA, AXB, and measurement at the linac for different heterogeneities

Table 2. Comparisons between AAA and Accuros XB algorithms for PTV

\begin{tabular}{cccc}
\hline \hline Parameter & AAA (mean \pm SD) & Accuros (mean \pm SD) & P \\
\hline $\mathrm{Cl}$ & $0.97 \pm 0.18$ & $0.99 \pm 0.14$ & 0.33 \\
$\mathrm{HI}$ & $0.11 \pm 0.01$ & $0.05 \pm 0.01$ & 0.04 \\
Dmax (Gy) & $69.30 \pm 3.12$ & $68.51 \pm 2.64$ & 0.02 \\
Dmean (Gy) & $66.30 \pm 0.65$ & $66.1 \pm 0.61$ & 0.54 \\
\hline \hline
\end{tabular}

Table 3. Comparisons between AAA and Acuros XB algorithms for OAR

\begin{tabular}{lcccc}
\hline \hline \multirow{2}{*}{ OAR } & \multicolumn{3}{c}{ AAA - Acuros XB (\%) } & \multirow{2}{*}{ P } \\
\cline { 2 - 4 } & Min Diff & Max Diff & Mean Diff & \\
\hline Spinal Cord & -6.3 & -1.3 & -2.5 & $<0.05$ \\
Heart D33 (Gy) & 2.4 & 8.2 & 4.9 & $<0.05$ \\
Heart D67 (Gy) & 0.7 & 7.2 & 3.3 & $<0.05$ \\
Heart D100 (Gy) & -1.02 & 1.9 & -0.4 & NS \\
Lung minus GTV D37 (Gy) & -6.3 & 1.1 & -2.4 & $<0.05$ \\
Lung minus GTV, Dmean (Gy) & -3.7 & -1.2 & -1.9 & $<0.05$ \\
\hline \hline
\end{tabular}




\section{Discussion}

The purpose of this work was to compare the dose calculated by two algorithms AAA and Acuros XB in a phantom with several heterogeneity at a given point in a first stage, and to expand the field of investigation in a second step to the clinical case, which presents a complex heterogeneity.

The algorithm AAA and the old algorithms have some limitations. The heterogeneous medium is converted to water and then the dose distribution is calculated. The Acuros XB algorithm calculates in the inhomogenous absorber using a list of materials and a look up table to describe the chemical composition of each voxel [22].

The result of our phantom study showed a good agreement with several studies that demonstrated the superiority of the Acuros XB over the AAA in predicting dose when it concerns heterogeneous media [23-25].In the presence of heterogeneity: water; lung; bone, the algorithm Acuros $\mathrm{XB}$ is more precise and very close to the measurements. The algorithm AAA underestimates the calculated dose beyond these heterogeneous medium while in the presence of the muscle the AAA overestimates the dose.

For the clinical study, the AAA and AXB plans were evaluated based on the results derived from the Dose Volume Histogram DVH in the Eclipse TPS. In this study, analysis of DVH for all 20 patients showed no significant differences for the mean dose and conformity index at the target volume ( $p>0.05$ ) for both algorithms (AAA and Acuros $\mathrm{XB})$. This result is similar to the results reported in many studies [26-29].

However, there was a statistically significant difference observed for the Homogeneity Index (HI) and Maximum Dose
(Dmax) to the target, between the two algorithms ( $p<0.05)$, the $\mathrm{HI}$ and Dmax for AAA were higher than AXB. Similar result was described in several publications [26, 14, 30, and 31].

For organs at risk, the results of the study carried out by Sterpin et al [32] is in alignment with our findings for AAA and Acuros XB calculations. As illustrated in Table 2, there is a statistical significant difference for all the organs $(p<0.05)$ except for the heart, where there is no significant difference for the dose received by $100 \%$ of the heart volume.

\section{Conclusion}

The results of our measurements phantom study showed a very good agreement with calculations carried out with Acuros algorithm. Little worse agreement was obtained for AAA algorithm.

The results of our clinical study showed no significant differences for the mean dose and conformity index at the target volume for both algorithms, however the Homogeneity Index and Maximum Dose are significantly different. For organs at risk, there is a statistical significant difference for the majority of organs.

\section{Acknowledgements}

We wish to thank all the medical staff of Sheikh Khalifa Ibn Zaid Hospital where the data acquisition was carried out for this study. In addition, we would like to express our sincere thanks to Dr. Guessous Fadila, Professor at Université Mohammed VI des Sciences de la Santé for her excellent support in this work.

\section{References}

[1] Clarkson JR. A note on depth doses in fields of irregular shape. Br J Radiol. 1941;14(164):265-268.

[2] Cunningham JR. Scatter-air ratios. Phys Med Biol.1972;17(1):42-51.

[3] Khan FM, Levitt SH, Moore VC, Jones TK Jr. Computer and approximation methods of calculating depth dose in irregularly shaped fields. Radiology. 1973;106(2):433-436.

[4] Sontag MR, Cunningham JR. The equivalent tissue-air ratio method for making absorbed dose calculations in a heterogeneous medium. Radiology. 1978;129(3):787-794.

[5] Webb S, Fox RA. Verification by Monte Carlo methods of a power law tissue-air ratio algorithm for inhomogeneity corrections in photon beam dose calculations. Phys Med Biol. 1980;25(2):225-240.

[6] Papanikolaou N, Battista J, Boyer A, et al. Tissue Inhomogeneity Corrections for Megavoltage Photon Beams. AAPM Report No. 85, AAPM TG65, 2004.

[7] Mohan R, Chui C, Lidofsky L. Differential pencil beam dose computation model for photons. Med Phys. 1986;13(1):64-73.

[8] Ahnesjö A. Collapsed cone convolution of radiant energy for photon dose calculation in heterogeneous media. Med Phys. 1989;16(4):577-592.

[9] Ahnesjö A, Saxner M, Trepp A. A pencil beam model for photon dose calculation. Med Phys. 1992;19(2):263-273.

[10] Bourland JD, Chaney EL. A finite-size pencil beam model for photon dose calculations in three dimensions. Med Phys. 1992;19(6):1401-1412.

[11] Ahnesjö A, Aspradakis MM. Dose calculations for external photon beams in radiotherapy. Phys Med Biol. 1999;44(11):R99-155.

[12] Knöös T, Wieslander E, Cozzi L, et al. Comparison of dose calculation algorithms for treatment planning in external photon beam therapy for clinical situations. Phys Med Biol. 2006;51(22):5785-5807. 
[13] Nakaguchi Y, Araki F, Maruyama M, Fukuda S. Comparison of RTPS and Monte Carlo dose distributions in heterogeneous phantoms for photon beams. Nihon Hoshasen Gijutsu Gakkai Zasshi. 2010;66(4):322-333.

[14] Han T, Mikell JK, Salehpour M, Mourtada F. Dosimetric comparison of Acuros XB deterministic radiation transport method with Monte Carlo and model-based convolution methods in heterogeneous media. Med Phys. 2011;38(5):2651-2664.

[15] Gagné IM, Zavgorodni S. Evaluation of the analytical anisotropic algorithm in an extreme water-lung interface phantom using Monte Carlo dose calculations. J Appl Clin Med Phys. 2007;8(1):33-46.

[16] Sievinen J, Ulmer W, Kaissl W. AAA photon dose calculation model in Eclipse. RAD \#7170B. Varian Medical Systems. 2005.

[17] Ulmer W, Kaissl W. The inverse problem of a Gaussian convolution and its application to the finite size of the measurement chambers/detectors in photon and proton dosimetry. Phys Med Biol. 2003;48(6):707-727.

[18] Ulmer W, Harder D. Applications of a Triple Gaussian Pencil Beam Model for Photon Beam Treatment Planning. Zeitschrift für Medizinische Physik. 1996;6(2):68-74.

[19] Ulmer W, Harder D. A Triple Gaussian Pencil Beam Model for Photon Beam Treatment Planning. Zeitschrift für Medizinische Physik. 1995;5(1):25-30.

[20] Vassiliev ON, Wareing TA, McGhee J, et al. Validation of a new grid-based Boltzmann equation solver for dose calculation in radiotherapy with photon beams. Phys Med Biol. 2010;55(3):581-598.

[21] IAEA-TECDOC-1540: Specification and Acceptance Testing of Radiotherapy Treatment Planning Systems. IAEA. 2007.

[22] Siebers JV, Keall PJ, Nahum AE, Mohan R. Converting absorbed dose to medium to absorbed dose to water for Monte Carlo based photon beam dose calculations. Phys Med Biol. 2000;45(4):983-995.

[23] Bush K, Gagne IM, Zavgorodni S, et al. Dosimetric validation of Acuros XB with Monte Carlo methods for photon dose calculations. Med Phys. 2011;38(4):2208-2221.

[24] Kan MW, Leung LH, So RW, Yu PK. Experimental verification of the Acuros XB and AAA dose calculation adjacent to heterogeneous media for IMRT and RapidArc of nasopharygeal carcinoma. Med Phys. 2013;40(3):031714.

[25] Han T, Mourtada F, Kisling K, et al. Experimental Validation of Deterministic Acuros XB Algorithm for IMRT and VMAT Dose Calculations with the Radiological Physics Center's Head and Neck Phantom. Med Phys. 2012;39(4):2193-2202.

[26] Fogliata A, Nicolini G, Clivio A, et al. Dosimetric evaluation of Acuros XB Advanced Dose Calculation algorithm in heterogeneous media. Radiat Oncol. 2011;6:82.

[27] Fogliata A, Nicolini G, Clivio A, et al. Critical appraisal of Acuros XB and Anisotropic Analytic Algorithm dose calculation in advanced non-small-cell lung cancer treatments. Int J Radiat Oncol Biol Phys. 2012;83(5):1587-1595.

[28] Kan MW, Leung LH, Yu PK. Dosimetric impact of using the Acuros XB algorithm for intensity modulated radiation therapy and RapidArc planning in nasopharyngeal carcinomas. Int J Radiat Oncol Biol Phys. 2013;85(1):e73-e80.

[29] Fogliata A, Scorsetti M, Navarria P, et al. Dosimetric comparison between VMAT with different dose calculation algorithms and protons for soft-tissue sarcoma radiotherapy. Acta Oncol. 2013;52(3):545-552.

[30] Rana S, Rogers K. Dosimetric evaluation of Acuros XB dose calculation algorithm with measurements in predicting doses beyond different air gap thickness for smaller and larger field sizes. J Med Phys. 2013;38(1):9-14.

[31] Robinson D. Inhomogeneity correction and the analytic anisotropic algorithm. J Appl Clin Med Phys. 2008;9(2):2786.

[32] Sterpin E, Tomsej M, De Smedt B, et al. Monte Carlo evaluation of the AAA treatment planning algorithm in a heterogeneous multilayer phantom and IMRT clinical treatments for an Elekta linear accelerator. Med Phys. 2007;34(5):1665-1677 